\title{
Which Computational Methods Are Good for Analyzing Large Photonic Crystal Membrane Cavities?
}

Malureanu, Radu; de Lasson, Jakob Rosenkrantz; Frandsen, Lars Hagedorn; Gutsche, Philipp; Burger, Sven; Kim, Oleksiy S.; Breinbjerg, Olav; Ivinskaya, Aliaksandra; Wang, Fengwen; Sigmund, Ole Total number of authors:

14

Published in:

Proceedings of the 20th Anniversary International Conference on Transparent Optical Networks

Link to article, DOI:

10.1109/ICTON.2018.8473951

Publication date:

2018

Document Version

Peer reviewed version

Link back to DTU Orbit

Citation $(A P A)$ :

Malureanu, R., de Lasson, J. R., Frandsen, L. H., Gutsche, P., Burger, S., Kim, O. S., Breinbjerg, O., Ivinskaya, A., Wang, F., Sigmund, O., Häyrynen, T., Lavrinenko, A., Mørk, J., \& Gregersen, N. (2018). Which Computational Methods Are Good for Analyzing Large Photonic Crystal Membrane Cavities? In Proceedings of the 20th Anniversary International Conference on Transparent Optical Networks IEEE. https://doi.org/10.1109/ICTON.2018.8473951

\section{General rights}

Copyright and moral rights for the publications made accessible in the public portal are retained by the authors and/or other copyright owners and it is a condition of accessing publications that users recognise and abide by the legal requirements associated with these rights.

- Users may download and print one copy of any publication from the public portal for the purpose of private study or research.

- You may not further distribute the material or use it for any profit-making activity or commercial gain

- You may freely distribute the URL identifying the publication in the public portal 


\title{
Which Computational Methods Are Good for Analyzing Large Photonic Crystal Membrane Cavities?
}

\author{
Radu Malureanu, Jakob Rosenkrantz de Lasson, Lars Hagedorn Frandsen, Philipp Gutsche ${ }^{\text {a }}$, Sven \\ Burger $^{\mathrm{a}}$, Oleksiy S. Kim ${ }^{\mathrm{b}}$, Olav Breinbjerg ${ }^{\mathrm{b}}$, Aliaksandra Ivinskaya ${ }^{\mathrm{c}}$, Fengwen Wang ${ }^{\mathrm{d}}$, Ole Sigmund ${ }^{\mathrm{d}}$, \\ Teppo Häyrynen, Andrei Lavrinenko, Jesper Mørk and Niels Gregersen \\ DTU Fotonik, Technical University of Denmark, Ørsteds Plads, Building 343, Kongens Lyngby, Denmark \\ Tel: (+45) 45253789, Fax: (+45) 45936581, e-mail: ngre@fotonik.dtu.dk \\ ${ }^{a}$ Zuse Institute Berlin (ZIB), Takustraße 7, D-14195 Berlin, Germany \\ ${ }^{b}$ DTU Elektro, Technical University of Denmark, Ørsteds Plads, Building 343, Kongens Lyngby, Denmark \\ ${ }^{c}$ ITMO University, Birzhevaja line, 14, 199034 St. Petersburg, Russia \\ ${ }^{d}$ DTU Mekanik, Technical University of Denmark, Ørsteds Plads, Building 343, Kongens Lyngby, Denmark
}

\begin{abstract}
By introducing defects into an otherwise periodic photonic crystal lattice, high quality (Q) factor cavities may be formed. However, the size and the lack of simplifying symmetries in the photonic crystal membrane make these types of cavities exceptionally hard to analyze using numerical simulation methods. In this work, we consider two different line defect cavities and we compute their Q factors using state-of-the-art optical simulation tools. We show that certain simulation methods perform much better than others in the analysis of these challenging structures.
\end{abstract}

Keywords: Photonic crystal, microcavity, line defect cavity, quality factor, numerical simulations.

\section{INTRODUCTION}

An important platform for planar optical integration is the photonic crystal [1] (PhC) membrane platform, where cavities and waveguides may play a key role in realizing compact optical components with classical functionality [2] such as switches, lasers, and amplifiers or quantum optical functionality such as integrated sources [3], [4] of quantum light. By leaving out a row of holes in an otherwise perfect $\mathrm{PhC}$ membrane lattice, a line defect is created in which light may be guided. If the waveguide is terminated at both ends, the finite-length waveguide forms an $\mathrm{L} n$ cavity, where $n$ denotes the length of the cavity. PhC cavities, and resonators more generally, are typically discussed in terms of their modes that, for example, show up as peaks in a scattering spectrum. In practical cavities, these peaks are broadened; the cavity mode dissipates power in the $\mathrm{PhC}$ and radiates power out of the $\mathrm{PhC}$, as quantified by the corresponding quality $Q$ factor proportional to the photon lifetime [5]. For laser applications, the $Q$ factor governs the onset of lasing, and for cavity quantum electrodynamics applications, it governs the onset of strong coupling. The $Q$ factor thus represents a key parameter in the design of a $\mathrm{PhC}$ membrane cavity [6].

However, the combination of the large size of the $\mathrm{PhC} \mathrm{L} n$ cavity and the full 3D nature of the geometry makes the calculation of the cavity $Q$ factor an extremely demanding numerical challenge [7]. No matter which numerical method is used, careful convergence checks [7] with respect to the degrees of freedom must be made. In this work, we focus on two structures, a low- $Q$ L5 cavity and a high- $Q$ L9 cavity. We employ the following five different computational methods [7] to compute the cavity $Q$ factor and the resonance wavelength $\lambda$ for both structures:

- The finite-difference time-domain (FDTD) technique.

- The finite-difference frequency-domain (FDFD) technique.

- Three variations of the finite-element method (FEM):

- An eigenvalue analysis based on JCMsuite (PFEM).

- A scattering analysis based on JCMsuite (sFEM).

- An eigenvalue analysis based on COMSOL (tFEM).

- The Fourier modal method (FMM).

- The surface integral equation (SIE) approach.

For each method, the relevant computational parameters are systematically varied to quantify the numerical error.

\section{PHOTONIC CRYSTAL MEMBRANE LINE DEFECT CAVITIES}

We consider a finite-size semiconductor PhC membrane geometry illustrated in Fig. 1 [7]. The structure is perforated by an in-plane triangular lattice of circular air cylinders and is surrounded by free space on all sides. The boundary of the structure is chosen such that the boundary cylinders are half circles as shown in Fig. 1. For the semiconductor material, we choose indium phosphide (InP), with refractive index $n_{\mathrm{InP}}$, that is also used in experiments with $\mathrm{PhC}$ membranes [8]. 


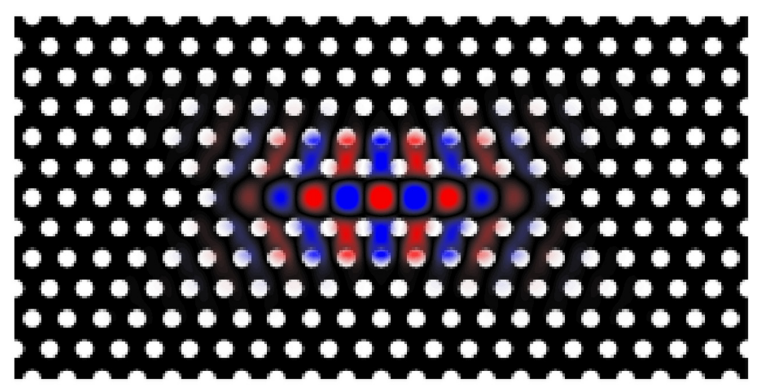

Fig. 1: Optical field $\left|E_{y}\right|^{2}$ profile for the fundamental L9 cavity mode.

According to MPB simulations [9], the periodic PhC structure exhibits a partial bandgap in the plane in the frequency range from 1372 to $1594 \mathrm{~nm}$, and by removing $n$ air holes centrally in the structure, an L $n$ cavity is formed. For two lengths of the cavity, $n=5$ and 9, we determine the wavelength and $Q$ factor of the fundamental mode in these cavities, the so-called M1 cavity mode, as function of computational parameters. Detailed structural parameters may be found in Ref. [7], and we stress that the surrounding air region is, in principle, of infinite extent.

The electric field profile of the fundamental M1 mode in the L9 cavity calculated with FMM is shown in Fig. 1, and the mode features an $E_{y}$ maximum at the center of the geometry. It is noted from Fig. 1 that the mode extends outside the cavity. For all five numerical methods, we verify qualitatively, by visual inspection, that the calculated modes have this field distribution. In this work, we focus on the convergence of $\lambda$ and $Q$ for the L5 and L9 configurations with parameters described in Ref. [7].

\section{NUMERICAL RESULTS}

While all methods employ an increasing number of degrees of freedom to provide a more accurate representation of the geometry and thus a more precise estimate of $\lambda$ and $Q$, the computational parameters used to describe the degrees of freedom vary greatly for the five methods. To enable comparison of the results in spite of the different parameters used by the methods, we thus present the results as function of a common geometrical setup index $j$ [7]. The exact parameters for the geometrical setup indices for each method are presented in Ref. [7], but different values of $j$ for FDTD and FMM, for example, correspond to different mesh sizes and Fourier truncations, respectively. Setup 1 contains the lowest number of degrees of freedom and the setup 8 the highest, and convergence for increasing setup index is thus expected. We use the term resolution broadly to discuss the performance of the methods when the number of degrees of freedom is varied.

\subsection{Smaller Structure and Shorter Cavity (L5)}

We initially focus on the shorter L5 cavity with in-plane PhC dimensions as given in Ref. [7]. For each of the five numerical techniques, we present calculations of the M1 wavelength and $Q$ factor as function of relevant

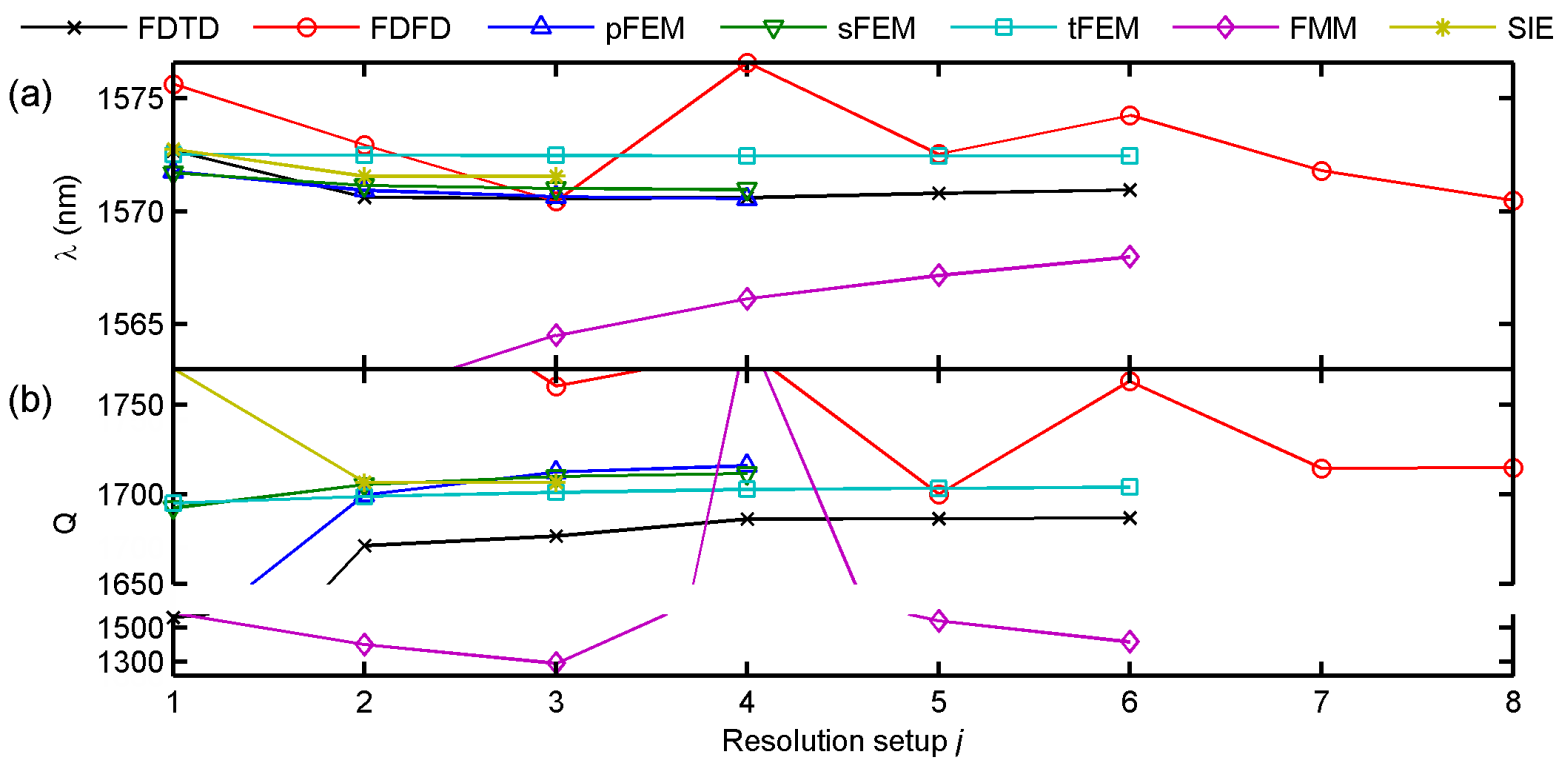

Fig. 2: L5: (a) Resonance wavelength $\lambda$ and (b) Q factor. Notice the disconnected y axis in (b). 
computational parameters.

In Fig. 2(a) we study the M1 resonance wavelength $\lambda$ as function of the resolution. The wavelength for the FDTD, pFEM, sFEM, tFEM and SIE methods converges fairly quickly towards a value of $\sim 1571 \mathrm{~nm}$. While the wavelength computed using the FDFD method converges more slowly, the final value is similar to that computed using the other methods. However, the wavelength for the FMM increases uniformly indicating that convergence has not been obtained.

The calculated $Q$ factor is presented in Fig. 2(b). Again the pFEM, sFEM, tFEM and SIE methods converge rapidly to values of $\sim 1710$, whereas the FDTD method converges towards a value of 1687 . Large variations are again observed for the FDFD technique, which finally converges towards a value similar to that of the pFEM, sFEM, tFEM and SIE methods. The FMM results feature the strongest variations and no clear convergence with a final $Q$ factor of 1417 representing a $\sim 20 \%$ error compared to the other methods.

\subsection{Larger Structure and Longer Cavity (L9)}

We now consider a longer L9 cavity that is expected to exhibit a higher $Q$ factor than the shorter cavity considered in the previous section. Also, the length and width of the PhC membrane are larger. As for the L5 cavity, we study the resonance wavelength and the $Q$ factor as function of the resolution setup.

The resonance wavelength $\lambda$ of the L9 cavity is presented in Fig. 3(a) as function of resolution setup. The overall behavior resembles that of the L5 cavity, the wavelength for the FDTD, the FEM and the SIE methods converges rapidly whereas the FDFD results display some variations. The FDTD, the FDFD, the FEM and the SIE methods produce similar wavelengths of $\sim 1578 \mathrm{~nm}$. In contrast, the final wavelength computed using FMM is $6 \mathrm{~nm}$ smaller, providing a first indication that the FMM has significant difficulty in handling the large L9 cavity.

Results for the $Q$ factor are presented in Fig. 3(b). The $Q$ factor from the FDTD, the FEM and the SIE methods converges towards $\sim 104,000$. Both the FDFD and the FMM methods display large variations, but whereas the FDFD results appear to converge slowly towards the value predicted by the other methods, the Q factor for the FMM oscillates around an average value of $\sim 25.000$ and thus deviates by almost a factor of 4 from all other results. The FMM thus appears as the least suitable method for handling the large L9 cavity.

\section{CONCLUSIONS}

The differences in the $Q$ factors and the resonance wavelengths obtained by the methods indicate that all methods have produced results deviating from the true ones to a larger or lesser degree [7]. While differences might be expected due to the use of different methods, our benchmark study of the 3 FEMs indicate that deviations occur even for different implementations of the same method.

In our study, independent simulations were performed by the different groups involved in this work using the computational hardware available to each group. In principle, this procedure minimizes the risk of systematic error, and the quantitative agreement may be regarded as more convincing as it has been reached independently. However, it does also mean that in the comparison we should keep in mind that different computational resources were available in the benchmarking of each method. Thus, while the change in computation time with the resolution setup can thus be compared for each method individually, when comparing the computation time

(a)

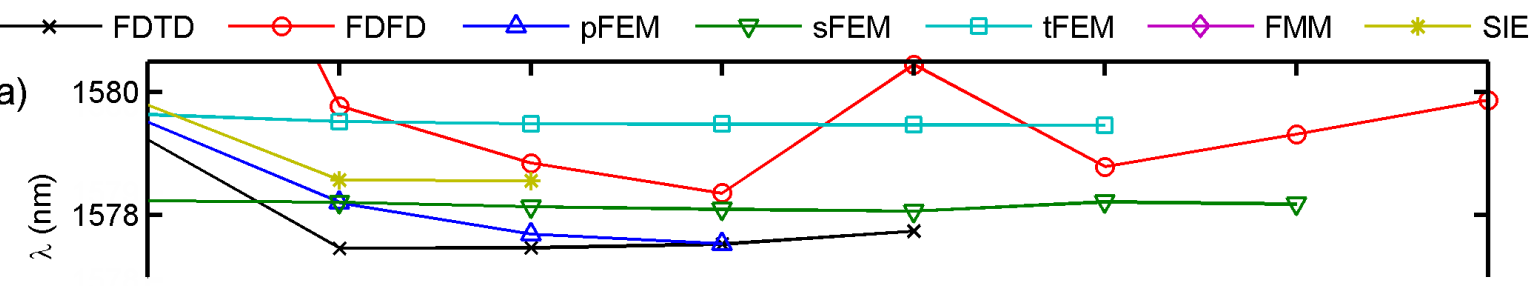

(b)

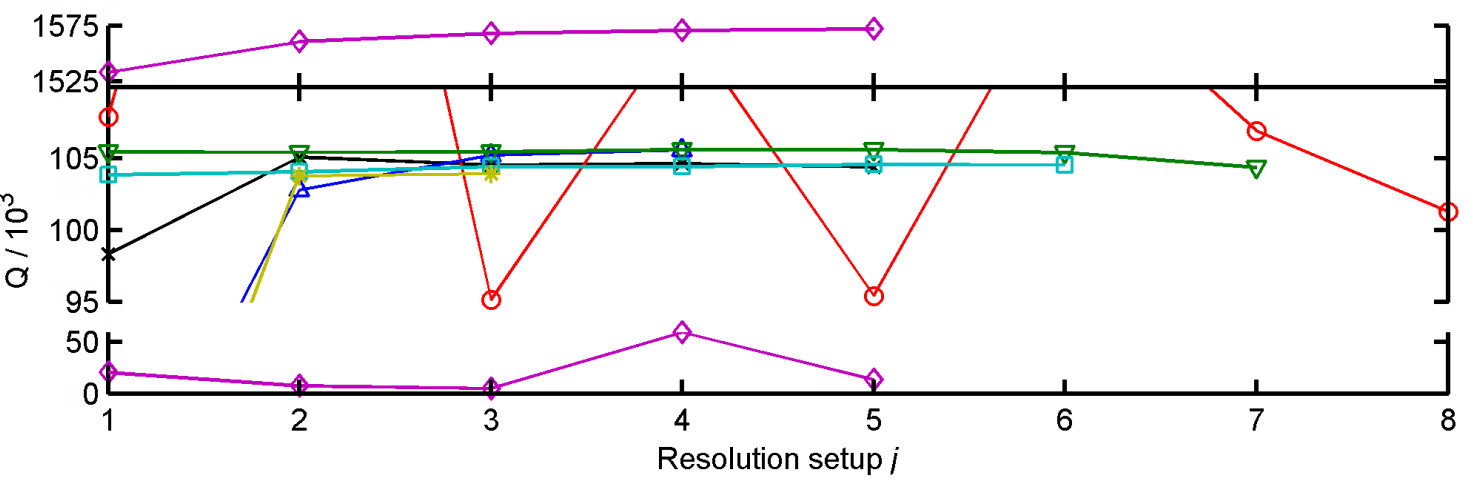

Fig. 3: L9: (a) Resonance wavelength $\lambda$ and (b) $Q$ factor. Notice the disconnected $y$ axis in (b). 
for the various methods one should keep in mind that different CPUs with varying clock frequencies were used. Keeping these differences in available resources in mind, our study [7] clearly indicates that the least suitable method for analyzing these large PhC line defect cavities is the FMM. The FMM is based on plane-wave expansion of the permittivity profile as well as of the electromagnetic fields, and the poor convergence is likely due to the difficulty of accurately describing the large electric field discontinuities occurring at the InP-air interfaces using this series expansion [10]. On the other hand, these field discontinuities are handled without any problem by the finite difference and finite elements methods which in this respect perform well, but at the cost of significant demands to memory, CPU cores and calculation time. The best performing method in our study is unarguably the SIE approach, which provides excellent convergence using the smallest memory requirement of all the methods [7].

\section{ACKNOWLEDGEMENTS}

We gratefully acknowledge funding from Villum Fonden (NATEC-II Center of Excellence), from the Danish Research Council for Technology and Production (Sapere Aude LOQIT, DFF-4005-00370), from the Einstein Foundation (ECMath) and from the German Research Foundation DFG (SFB787-B4).

\section{REFERENCES}

[1] J. D. Joannopoulos, S. G. Johnson, J. N. Winn, and R. D. Meade, Photonic Crystals. Molding the Flow of Light, 2nd ed. Princeton University Press, 2008.

[2] M. Notomi, A. Shinya, K. Nozaki, T. Tanabe, S. Matsuo, E. Kuramochi, T. Sato, H. Taniyama, and H. Sumikura, "Low-power nanophotonic devices based on photonic crystals towards dense photonic network on chip," IET Circuits, Devices Syst., vol. 5, no. 2, pp. 84-93, 2011.

[3] V. S. C. Manga Rao and S. Hughes, "Single quantum-dot Purcell factor and $\beta$ factor in a photonic crystal waveguide," Phys. Rev. B, vol. 75, no. 20, p. 205437, 2007.

[4] G. Lecamp, P. Lalanne, and J. P. Hugonin, "Very Large Spontaneous-Emission $\beta$ Factors in PhotonicCrystal Waveguides," Phys. Rev. Lett., vol. 99, no. 2, p. 23902, 2007.

[5] J. D. Jackson, "Waveguides, Resonant Cavities, and Optical Fibers," in Classical Electrodynamics, 3rd ed., Wiley, 1998, pp. 352-406.

[6] P. Lalanne, C. Sauvan, and J. P. Hugonin, "Photon confinement in photonic crystal nanocavities," Laser Photon. Rev., vol. 2, no. 6, pp. 514-526, 2008.

[7] J. R. de Lasson, L. H. Frandsen, P. Gutsche, S. Burger, O. S. Kim, O. Breinbjerg, A. Ivinskaya, F. Wang, O. Sigmund, T. Häyrynen, A. V. Lavrinenko, J. Mørk, and N. Gregersen, "Benchmarking five numerical simulation techniques for computing resonance wavelengths and quality factors in photonic crystal membrane line defect cavities," Opt. Express, vol. 26, no. 9, pp. 11366-11392, Apr. 2018.

[8] W. Xue, Y. Yu, L. Ottaviano, Y. Chen, E. Semenova, K. Yvind, and J. Mørk, "Threshold Characteristics of Slow-Light Photonic Crystal Lasers," Phys. Rev. Lett., vol. 116, no. 6, p. 63901, Feb. 2016.

[9] S. Johnson and J. Joannopoulos, "Block-iterative frequency-domain methods for Maxwell's equations in a planewave basis," Opt. Express, vol. 8, no. 3, pp. 173-190, Jan. 2001.

[10] H. S. Sözüer, J. W. Haus, and R. Inguva, "Photonic bands: Convergence problems with the plane-wave method," Phys. Rev. B, vol. 45, no. 24, pp. 13962-13972, Jun. 1992. 